\title{
An Assessment of Epidemiologic Burden of Hospitalization for Bronchial Asthma in Acute Exacerbation among Adult Patients Admitted in a Tertiary Hospital in the Philippines: A Pilot Study
}

Mithi Kalayaan S. Zamora, MD and Lenora C. Fernandez, MD

Division of Pulmonary Medicine, Department of Medicine, Philippine General Hospital, University of the Philippines Manila

\begin{abstract}
Objective. This pilot study aimed to determine the epidemiologic burden of hospitalization for bronchial asthma in acute exacerbation.

Methods. We used a descriptive cross-sectional study to describe the demographic profile, comorbidities and level of control of patients admitted for bronchial asthma in acute exacerbation admitted to the Philippine General Hospital from May 1 to October 31 2019. The study computed for the admission rate and compared the average cost of hospitalization (diagnostics, therapeutics and room) using direct costing to the total reimbursable cost from PhilHealth. Associations between patient characteristics (age, type of admission) and hospitalization cost were also determined.
\end{abstract}

Results. We enrolled 45 patients in the study. The admission rate was $8 /$ month. The mean cost of admission was significantly higher than the reimbursable amount from PhilHealth (Php 20,074.63 versus Php 9000) $(p=0.004)$. Cost of hospitalization was significantly higher in pay patients versus charity $(p=0.001$ for diagnostics, $p=0.005$ for treatment and $p=0.001$ for room cost), in patients with poorly controlled asthma ( $p=0.020$ for diagnostics and $p=0.014$ for room cost) and those with frequent short-acting beta-agonist (SABA) use $(p=0.001)$.

Conclusion. Asthma remains an economic burden for the Filipino patient. Persistent SABA use, perceived asthma control of patients and increased expenses associated with an asthma exacerbation admission lead to significant out-of-pocket expenditure.

Key Words: bronchial asthma, exacerbation, direct costing

\section{INTRODUCTION}

Asthma is a controllable but chronic disease affecting people from all walks of life. Left uncontrolled, it is debilitating and may lead to hospitalization, absence from school and/or work and lost income or opportunity cost. Multiple factors affect risk of exacerbation which could potentially lead to hospitalization with out-of-pocket cost.

Through the case rate system, PhilHealth aims to Corresponding author: Mithi Kalayaan S. Zamora, MD Division of Pulmonary Medicine Department of Medicine Philippine General Hospital University of the Philippines Manila Taft Avenue, Manila 1000 Philippines Email: mithikalayaanzamora@gmail.com expand population coverage, increase benefits and change the provider system to reduce out-of-pocket payments. For asthmatic patients admitted at a PhilHealth-accredited health institution, this ideally ensures that hospitalizations from exacerbations are subsidized by the government with minimal to almost no additional expenses incurred. 
However, according to the Philippine Institute for Development Studies (PIDS), despite efforts by our government, out-of-pocket medical expense in the Philippines remains high. ${ }^{1}$ In 2013, Filipino households spent P296.5 billion for out-of-pocket health expenses. ${ }^{1}$

At present, no local data is available to determine outof-pocket expenditure of patients admitted for bronchial asthma in exacerbation or patient characteristics that increased risk of admission from exacerbation.

\section{Asthma as a Public Health Problem}

Asthma is a global public health problem. According to the World Health Organization, the human and economic burden of asthma is severe, with economic costs exceeding those of TB and HIV/AIDS combined. ${ }^{2}$ Asthma is defined as a heterogenous disease associated with chronic airway inflammation. ${ }^{3}$ It is associated with respiratory symptoms such as wheezing, shortness of breath, chest tightness and cough that vary over time and intensity, together with variable expiratory flow limitation. ${ }^{3}$ A local Philippine study done in 2014 showed an overall adult asthma prevalence of $14.3 \%$ with greater prevalence of asthma among males. ${ }^{4}$

\section{Cost of Asthma Care}

A 2016 study explored asthma cost and economic burden, including all disease-related costs - direct cost including asthma management, indirect cost including workrelated losses, and intangible loss including decreases in quality of life. ${ }^{5}$ The mean cost per patient per year of asthma exacerbation amounted to $\$ 1,900$ (or P98,800) in Europe and an estimated mean of $\$ 3,100$ (or $\mathbf{P} 161,200$ ) in the US. In the same study, ${ }^{5}$ factors that increased cost included high severity or uncontrolled asthma, low income, multiple comorbidities and advanced age.

\section{Universal Health Care}

The National Health Insurance Program (PhilHealth) was established to provide health insurance coverage and ensure affordable, acceptable, available and accessible health care services for all citizens of the Philippines. ${ }^{6}$ During the Aquino administration, the Department of Health through Administrative Order No 2010-0036 launched "Kalusugang Pangkalahatan" aiming to achieve universal health care for all Filipinos. ${ }^{7}$ In agreement with "Kalusugang Pangkalahatan," the case rate payment scheme was initiated. ${ }^{8-10}$ In addition to the case rate payment scheme, the No Balance Billing Policy was employed for sponsored, indigent and special members (kasambahay, senior citizen) admitted at charity beds in government hospitals. ${ }^{8-10}$ Through this policy changes, PhilHealth aimed to expand population coverage, increase benefits and change the provider system to reduce out-of-pocket payments. ${ }^{11}$

To the author's knowledge, no local published study has yet identified out-of-pocket medical expense for bronchial asthma in acute exacerbation nor determined its economic burden.
In this study, we aimed to determine the cost of hospitalization of adult patients admitted for asthma in acute exacerbation. The estimated cost was compared to the case rate provided by PhilHealth to determine the adequacy of subsidy. In addition, the study aimed to describe the demographic profile and comorbidities of admitted patients, determine associations between patient characteristics and hospitalization cost, and determine associations between perceived and actual level of control with exacerbation.

\section{OBJECTIVES}

This study aimed to determine the following:

1. rate of admission for acute bronchial asthma exacerbation

2. hospitalization cost

3. perceived level of control and use of asthma medications

4. associations between these variables

\section{METHODS}

We conducted a descriptive cross-sectional pilot study among patients admitted with bronchial asthma in acute exacerbation at the Philippine General Hospital from May 1 to October 31, 2019.

\section{Inclusion Criteria}

1. Admissions from both charity and pay wards of adult patients $\geq 19$ years old

2. Main diagnosis of bronchial asthma and/or PhilHealth enrollment claims using the ICD-10-CM Diagnosis Code J45.901

\section{Exclusion Criteria}

1. Admissions from both charity and pay wards of adult patients $\leq 18$ years old

2. Primary assessment is not bronchial asthma in acute exacerbation and/or ICD-10-CM Diagnosis Code J45.901

3. Unstable comorbidities

4. Admitting diagnosis with comorbidities containing case-rates greater than bronchial asthma in acute exacerbation

5. Lack of consent for participation in the study

Since this is a pilot study, no sample size was set, and instead, all patients admitted during the study period who fulfilled the inclusion criteria were invited to participate in the study. Demographic profile and direct health cost of admitted patients for asthma exacerbation at UP-PGH for six months were extracted using chart review and patient interview. Regular review of ward reports from both charity and pay services was done to verify admissions. Patients were approached personally by the primary investigator and/or research assistant with a brief introduction to the research - title, general objectives and inclusion criteria. 
Potential patient participants were given consent forms for their review. Once consent was secured, the patient was interviewed and the chart was reviewed. The interview lasted for a maximum of ten minutes.

A data extraction form was used during the chart review which included baseline characteristics of the patients, demographics, comorbidities, and length of hospital admission. An interview form was used to evaluate asthma control (as perceived by the patient and as evaluated by the primary investigator and/or research assistant) including prior hospitalizations and a list of maintenance medications. Perception of asthma control by the patient was subjectively asked while evaluation of control by the primary investigator and/or research assistant was objectively determined using the Global Initiative for Asthma (GINA) guide.

Direct cost of diagnostics, therapeutics and room rate was estimated and compared to the reimbursable cost from PhilHealth. Since there were no billing statements available in PGH, chart review was done to check medications given and laboratory tests done. The therapeutic sheet was reviewed to assess if medications were administered, and doses were converted to preparations available at the pharmacy and number of products consumed. Official laboratory results and/or chart documentation of results of diagnostics were counted including the number of times a particular laboratory test was requested.

Cost of diagnostic procedures was requested from the hospital through the Department of Laboratories while cost of medications was requested from the hospital pharmacy. Costing for both charity and pay was standardized and was assumed using the Class B rates, the charity rate with the highest price. The room costing for pay patients was determined by the room rate. Since the case rate system includes medications and laboratories requested regardless of, and unrelated to the main diagnosis, all requested diagnostics and therapeutics even of workups and treatment of comorbidities were included in the total costing.

We used descriptive statistics, ANOVA and multiple linear regression to analyze the data extracted to determine associations between patient characteristics and cost of asthma exacerbation.

\section{RESULTS}

A total of 45 patients who fulfilled the inclusion criteria were included during the 6-month study period.

An average of 2 patients were admitted every week or 8 patients every month $(\mathrm{n}=45)$ for asthma in exacerbation, with most admissions belonging to charity (73.3\%).

Most of the patients who were admitted for bronchial asthma in acute exacerbation were aged 36-45 years (33.3\%), female (28, 62.2\%), from the Greater Manila Area (Cavite, Laguna) and were admitted at the charity wards $(33,73.3 \%)$. Around a quarter $(n=13,28.9 \%)$ of patients admitted had hypertension as a comorbidity.
Majority ( $\mathrm{n}=40,88.9 \%)$ of patients admitted with a primary working impression of asthma in acute exacerbation while 3 were cardiac diagnosis, 1 was infectious and 1 was surgical. There were three mortalities due to acute myocardial infarction (2) and septic shock from hospital acquired pneumonia (1). Sixty percent (27) of all patients were admitted for $>7$ days, $33.3 \%$ (15) were admitted for 3-7 days and $6.7 \%$ (3) were admitted for $<3$ days.

More than half of patients evaluated themselves to have controlled asthma (54.8\%) while the physician interviewer only assessed one third (31\%) of patients to have controlled asthma. Majority of patients were on short-acting betaagonist (SABA) pro re nata (PRN). 3 patients refused to answer their perception of control of their asthma preadmission including compliance to maintenance medications and refused to give information as well regarding objective parameters for the primary investigator/research assistant to evaluate level of asthma control as well (Table 1).

Table 1. Clinical characteristics of patients $(N=45)$

\begin{tabular}{lrc}
\multicolumn{1}{c}{ Characteristic } & No. & $\%$ \\
Asthma control prior to admission (by physician) & & \\
Controlled & 13 & 31.0 \\
Partly controlled & 12 & 28.6 \\
Uncontrolled & 17 & 40.5 \\
\hline Asthma control prior to admission (by patient) & & \\
Controlled & 23 & 54.8 \\
Partly controlled & 10 & 23.8 \\
Uncontrolled & 9 & 21.4 \\
\hline Pre-admission maintenance medication & \multicolumn{2}{c}{} \\
SABA PRN & 40 & 88.8 \\
LABA + Long acting ICS & 0 & 0.00 \\
Others & 5 & 11.1 \\
\hline Compliance to medications as assessed by patient & & \\
Good compliance & 34 & 81.0 \\
Fair compliance & 3 & 7.1 \\
Uncompliant & 5 & 11.9 \\
\hline
\end{tabular}

SABA, Short-acting beta-agonist; PRN pro re nata; LABA, Long-acting beta-agonist; ICS, inhaled corticosteroids

On average, a patient spent Php 7,476.36 for diagnostics, Php 9,903.00 for treatment and Php 2,710.67 for room cost during an admission for exacerbation (Table 2).

Table 2. Cost of hospitalization (Php) based on type of cost

\begin{tabular}{ccrrr} 
Type of Cost & Mean & \multicolumn{1}{c}{ SD } & Minimum & Maximum \\
\hline Diagnostics & 7476.36 & 3417.20 & 180.00 & 16553.00 \\
Treatment & 9903.00 & 17631.43 & 839.75 & 72546.25 \\
Room & 2710.67 & 5461.98 & 0.00 & 18560.00 \\
\hline
\end{tabular}

Using simple linear regression, the biggest contributor to the total direct cost of admission was the diagnostic cost (every unit increase leads to a 6.93 increase in total cost), followed by room cost (4.64 increase in total cost), and treatment cost (1.65 increase in total cost).

There was no significant difference in the average costs between various age groups (Table 3 ). 
Table 3. Comparison of cost of hospitalization (Php) based on age (yrs.)

\begin{tabular}{|c|c|c|c|c|c|c|c|c|}
\hline Type of Cost & $19-25$ & $26-35$ & $36-45$ & $46-55$ & $56-65$ & $>65$ & Test-Statistic & P-Value* \\
\hline Total cost & 10237.50 & 16201.50 & 33856.08 & 11110.75 & 15714.40 & 14532.50 & N/A & N/A \\
\hline Diagnostics & 7320.00 & 8840.00 & 9028.83 & 7317.71 & 5379.36 & 6983.25 & 1.506 & 0.21 \\
\hline Treatment & 2917.50 & 7361.50 & 20061.95 & 3403.71 & 7675.04 & 2749.25 & 1.712 & 0.16 \\
\hline Room & 0.00 & 0.00 & 4812.00 & 333.33 & 2660.00 & 4800.00 & 1.247 & 0.31 \\
\hline
\end{tabular}

${ }^{*}$ ANOVA

There was a significant difference in the mean total hospital cost for the 36-45, 46-55, 56-65 year-old age groups compared to the reimbursable amount from PhilHealth (Appendix). The test was not conducted to the first and second age group since only 1 respondent answered in each group.

The mean costs for diagnostics, treatment and room were lower for charity than pay (Table 4), and mean total cost (pay, Php 42,120.52; charity, Php 12,057.93) for both were significantly higher than the reimbursable amount from PhilHealth (Appendix).

Table 4. Comparison of mean cost of hospitalization (Php) based on location

\begin{tabular}{ccrcc} 
Type of Cost & Pay & Charity & Test-Statistic & P-Value $^{*}$ \\
Diagnostics & 10105.72 & 6520.23 & 3.481 & 0.001 \\
Treatment & 21849.81 & 5559.71 & 2.976 & 0.005 \\
Room & 10165.00 & 0.00 & 9.845 & 0.001 \\
\hline
\end{tabular}

*Independent sample t-test

There was a statistically significant difference in cost for diagnostics and room based on the level of asthma control prior to admission. However, treatment cost does not differ between levels of asthma control (Table 5). The mean total cost was significantly higher for controlled and partially controlled asthma compared to the reimbursable amount from PhilHealth, but not significantly different for uncontrolled asthma (Appendix).

Table 5 shows the ANOVA test of the different types of cost per level of asthma control. From the table, there is statistically significant difference in cost for diagnostics and room based on the level of asthma control prior to admission. In comparison, treatment cost does not differ between levels of asthma control.
A simple linear regression was done to determine effect of each cost to the total cost of hospitalization. From the analysis, every unit increase in the diagnostic cost leads to a 6.925 increase in total cost, a unit increase in therapeutic cost leads to a 1.6494 increase in total cost and a unit increase in room cost leads to a 4.6385 increase in total cost. With this, we can see that the biggest contributor to the total direct cost of admission comes from the diagnostics done during an admission.

From Table 6, the average patient who gets admitted spends Php 20,074.63 for an admission for bronchial asthma in acute exacerbation. The current case rate for the same illness covered by PhilHealth as of December 2019 is currently at Php 9,000.00. Using one sample t-test, a $\mathrm{p}$-value of 0.004 was determined which signifies that there is significant difference in the average hospital cost and rate given by PhilHealth.

Statistically, there is a moderate negative correlation between asthma control and maintenance medications used pre-admission. Since the p-value is 0.001 which is less than 0.05 with a correlation coefficient of -0.506 , it translates clinically that as the medicine become more effective the more the asthma becomes uncontrollable. From the data gathered and interview of patients, none of the patients admitted were using LABA + ICS and majority were using SABA PRN for symptomatic relief.

A correlation between asthma control and medications prior to discharge was also made statistically. The hypothesis was that increased maintenance medications prior to discharge is correlated with decreasing control of asthma. However, there is no correlation between asthma control and discharge medications from the statistical analysis (correlation coefficient 0.025 ; $\mathrm{p}$-value 0.874 ).

Table 5. Comparison of cost of hospitalization (Php) based on level of asthma control

\begin{tabular}{lrcccc} 
Type of Cost & Controlled & Partly controlled & Uncontrolled & Test-Statistic & P-Value* \\
\hline Diagnostics & 8896.00 & 5894.30 & 5918.33 & 4.341 & 0.020 \\
Treatment & 13009.57 & 9342.19 & 5472.55 & 0.576 & 0.567 \\
Room & 5129.57 & 0.00 & 444.44 & 4.755 & 0.014 \\
\hline${ }^{*}$ ANOVA & &
\end{tabular}

Table 6. Descriptive Statistics of the Total Hospitalization Cost for Asthma Patients

\begin{tabular}{ccccc} 
Average & Standard Deviation & Minimum & Maximum & p-value \\
20074.63 & 24527.45 & 1019.75 & 107659.25 & 0.004 \\
\hline
\end{tabular}




\section{DISCUSSION}

Asthma remains a public health problem in both developed and developing countries affecting all walks of life. ${ }^{1}$ Despite the availability of effective medications for asthma, patients still get admitted for exacerbation.

The admission rate (2 patients/week or 8 patients/month) for asthma in exacerbation, with majority of admissions in the charity division, reflected the findings of the REALISE Asia study in $2015 .{ }^{11}$ This shows that there remains a gap between the perceived control among patients and healthcare providers, which is also correlated with the increased incidence of admission for exacerbation. Despite efforts to educate patients with asthma regarding the appropriate medication for asthma (LABA+ICS), a significant majority still prefer the immediate relief provided by as-needed SABA claiming good compliance with this identified "maintenance medication" for asthma.

Although almost all patients get discharged after an admission, there is prolonged hospital stay which could possibly be confounded by the decompensation of the patients' comorbidities resulting to increased hospitalization cost. In the study, the death of three patients were from another cause different from the reason for admission.

The main objective of the study was to determine cost of hospitalization of bronchial asthma in acute exacerbation using direct costing and subsequently comparing this costing to the case rates provided by the National Health Insurance Program (PhilHealth). Two studies in 20159,10 already evaluated that the out-of-pocket medical expense in the Philippines is still high. Results from the study confirm that this still holds true in 2019, with an average patient spending Php 20,090.03 for an admission while the current Metro Manila minimum wage (as of Nov 2018) is Php 537/day or Php 11,814 for a 22-day work month and the current case rate coverage for asthma in acute exacerbation is Php 9,000.

Descriptive statistics of the total hospitalization cost for asthma patients in comparison to the case rate for asthma in exacerbation show a significant difference between the average hospital cost and coverage provided by PhilHealth. Reasons for this gap include 1) the presence of comorbidities of the patients initially admitted for asthma exacerbation resulting in additional diagnostics and prolonged hospitalization and 2) increasing cost of healthcare in the Philippines.

Pay patients were noted to have increase out-of-pocket expense than charity patients even after adjustments were made standardized. This is assumed to be due to 1) lack of additional cost for room on charity basis and 2) perceived limitations in the resources at the charity resulting to fewer diagnostics and slower turnover of laboratories ordered. However, age was not correlated with increased cost of hospitalization.

Another result from the study was the effect of level of asthma control with the diagnostics and room cost. With increasing perceived of lack of control from the health provider, there is a tendency to order more tests and this results to the increased hospitalization days and corollary to it, an increase in the cost of room. However, it was also shown from the data correlation that there is no association between level of asthma control prior to admission and the cost of treatment. This is primarily because management of asthma in acute exacerbation remains standardized and most clinicians managing these cases give the same medications upon admission - steroids and SABA nebulization, regardless of the history of level of control prior to admission.

Finally, maintenance medications prior to admission and upon discharge were correlated with asthma control to see association between asthma control and medications - which could have affected direct costing upon admission and could potentially affect expenses post discharge from the hospital. The data showed a moderate negative correlation between increased use of SABA (which patients perceived as more efficacious maintenance medication) with uncontrolled asthma that may have resulted in increased hospitalization cost. However, type of medications prior to discharge was not correlated with asthma control.

\section{CONCLUSION}

Asthma remains an economic burden for all patients. The immediate relief provided by SABA PRN together with the perceived level of control of asthmatic patients all lead to an increased risk and duration of hospitalization. Patients admitted at the pay wards, on frequent SABA use, multiple uncontrolled comorbidities and with perceived lack of control from the health care provider may increase cost for hospitalization. At present, the current coverage provided by the national insurance claims in our country for hospitalization remain inadequate to minimize out-ofpocket expenditure among patients admitted for bronchial asthma in acute exacerbation. And while there is room for public health information campaigns on the proper management and control of asthma, the economic cost and subsequently, coverage for asthma may need to be revisited to provide adequate coverage for our Filipino patients who get admitted for an exacerbation.

\section{Limitations of the study}

Due to unavailability of billing statements, other health facility expenses including cost of supplies and personnel (including professional fee) were excluded and viewed as a limitation in the computation of cost. Severity of exacerbation was not assessed, which could have affected direct costing. In addition, the study only computed for direct costing and not opportunity cost .

The SABA use was attributed to the behavior of the patients claiming good compliance due to immediate relief. However, behavior of attending physicians regarding medication prescription was not evaluated which could possibly affect patients' perception on SABA use. 


\section{Statement of Authorship}

Both authors participated in the analysis and approval of the final version submitted.

\section{Author Disclosure}

Both authors declared no conflicts of interest.

\section{Funding Source}

This study received funding from the University of the Philippines - Philippine General Hospital Research Grant for 2019.

\section{REFERENCES}

1. Picazo O,Ulep VGT,Pantig IM,Ho BL.A critical analysis of purchasing of health services in the Philippines: a case study of PhilHealth. 2015 [Internet]. [cited 2015]. Available from: https://dirp3.pids. gov.ph/websitecms/CDN/PUBLICATIONS/pidsdps1554.pdf.

2. World Health Organization: Bronchial Asthma Fact Sheets [Internet]. [cited 2018 Aug]. Available from: http://www.who.int/mediacentre/ factsheets/fs206/en/

3. Global Initiative for Asthma. Global Strategy for Asthma Management and Prevention (2018 Update) [Internet]. [cited 2018 Sep]. Available from: https://ginasthma.org/wp-content/uploads/2018/04/wmsGINA-2018-report-tracked_v1.3.pdf
4. Varona LL, Alava HDA, Abong JM, Castor MAR, De Leon JC, Kwong SL. Prevalence of asthma among Filipino adults based on the National Nutrition and Health Survey (NNHeS). Philipp J Intern Med. 2014 Oct-Dec; 52(4):1-7.

5. Nunes C, Pereira A, Morais-Almeida M. Asthma costs and social impact. Asthma Res Pract. 2017; 3:1. doi: 10.1186/s40733-0160029-3.

6. PhilHealth Agency's Mandate and Functions [Internet]. [cited 2014 Aug]. Available from: https://www.philhealth.gov.ph/about_us/ mandate.html.

7. Department of Health. National Objectives for Health 2011-2016. July 2012.pp.132.

8. Philippine Health Insurance Corporation. New case rate packages from PhilHealth [Internet. [cited 2011 Aug 18]. Available from: http://www.philhealth.gov.ph/news/2011/new_caserates.php.

9. Philippine Health Insurance Corporation. PhilHealth Circular No. 011-A-2011 Selected medical case-rates - additional implementing guidelines [Internet]. [cited 2011 Aug 18]. Available from: http:// www. philhealth.gov.ph/circulars/2011/circ11-A_2011.pdf.

10. Philippine Health Insurance Corporation. PhilHealth Circular No. 2017-006 Strengthening the Implementation of the No Balance Billing Policy (Revision 1) [Internet]. [cited 2017 Jan 24]. Available from: https://www.philhealth. gov.ph/circulars/2017/ circ2017-0006.pdf.

11. Price D, David-Wang A, Cho SH, Ho JC, Jeong JW, Liam CK, et al. Time for a new language for asthma control: results from REALISE Asia. J Asthma Allergy. 2015 Sep; 8:93-103. doi: 10.2147/ JAA.S82633.

\section{APPENDIX}

Appendix 1. Mean total hospital cost based on location and level of asthma control compared with the current case rate for asthma in acute exacerbation
Characteristic
Mean cost (Php)
Test statistic
P-value*

\begin{tabular}{llll} 
Age group (yrs.) ${ }^{\dagger}$ & & & \\
$36-45$ & 33856.08 & 2.469 & .027 \\
$46-55$ & 11110.75 & 6.413 & .000 \\
$56-65$ & 15714.40 & 2.866 & .019 \\
$\quad$ Older than 65 & 14532.50 & 1.228 & .307 \\
\hline Location & & & \\
Pay & 42120.52 & 2.868 & .015 \\
$\quad$ Charity & 12057.93 & 3.281 & .002 \\
\hline Level of asthma control & & & \\
Controlled & 27035.13 & 2.656 & .014 \\
Partially controlled & 15236.49 & 3.573 & .006 \\
Uncontrolled & 11758.33 & 1.110 & .299 \\
\hline
\end{tabular}

*one-sample t-test; 'younger age groups were not analyzed since only 1 patient per age group $\dagger$ 